\title{
Campylobacter jejuni and Campylobacter coli autotransporter genes exhibit lineage- associated distribution and decay
}

\author{
Jai W. Mehat ${ }^{*}$ D, Roberto M. La Ragione and Arnoud H. M. van Vliet ${ }^{*}$
}

\begin{abstract}
Background: Campylobacter jejuni and Campylobacter coli are major global causes of bacterial gastroenteritis. Whilst several individual colonisation and virulence factors have been identified, our understanding of their role in the transmission, pathogenesis and ecology of Campylobacter has been hampered by the genotypic and phenotypic diversity within C. jejuni and C. coli. Autotransporter proteins are a family of outer membrane or secreted proteins in Gram-negative bacteria such as Campylobacter, which are associated with virulence functions. In this study we have examined the distribution and predicted functionality of the previously described capC and the newly identified, related capD autotransporter gene families in Campylobacter.

Results: Two capC-like autotransporter families, designated capC and capD, were identified by homology searches of genomes of the genus Campylobacter. Each family contained four distinct orthologs of CapC and CapD. The distribution of these autotransporter genes was determined in 5829 C. jejuni and 1347 C. coli genomes. Autotransporter genes were found as intact, complete copies and inactive formats due to premature stop codons and frameshift mutations. Presence of inactive and intact autotransporter genes was associated with $C$. jejuni and $C$. coli multi-locus sequence types, but for $c a p C$, inactivation was independent from the length of homopolymeric tracts in the region upstream of the capC gene. Inactivation of capC or capD genes appears to represent lineagespecific gene decay of autotransporter genes. Intact capC genes were predominantly associated with the C. jejuni ST-45 and C. coli ST-828 generalist lineages. The capD3 gene was only found in the environmental C. coli Clade 3 lineage. These combined data support a scenario of inter-lineage and interspecies exchange of cap $C$ and subsets of capD autotransporters.
\end{abstract}

Conclusions: In this study we have identified two novel, related autotransporter gene families in the genus Campylobacter, which are not uniformly present and exhibit lineage-specific associations and gene decay. The distribution and decay of the capC and capD genes exemplifies the erosion of species barriers between certain lineages of C. jejuni and C. coli, probably arising through co-habitation. This may have implications for the phenotypic variability of these two pathogens and provide opportunity for new, hybrid genotypes to emerge.

Keywords: Campylobacter, Jejuni, Coli, Autotransporter proteins, Genomics, Recombination

*Correspondence: jw.mehat@surrey.ac.uk; a.vanvliet@surrey.ac.uk Department of Pathology and Infectious Diseases, School of Veterinary Medicine, University of Surrey, Guildford, UK

C C The Author(s). 2020 Open Access This article is licensed under a Creative Commons Attribution 4.0 International License, which permits use, sharing, adaptation, distribution and reproduction in any medium or format, as long as you give appropriate credit to the original author(s) and the source, provide a link to the Creative Commons licence, and indicate if changes were made. The images or other third party material in this article are included in the article's Creative Commons licence, unless indicated otherwise in a credit line to the material. If material is not included in the article's Creative Commons licence and your intended use is not permitted by statutory regulation or exceeds the permitted use, you will need to obtain permission directly from the copyright holder. To view a copy of this licence, visit http://creativecommons.org/licenses/by/4.0/ The Creative Commons Public Domain Dedication waiver (http://creativecommons.org/publicdomain/zero/1.0/) applies to the data made available in this article, unless otherwise stated in a credit line to the data. 


\section{Background}

Campylobacter jejuni and Campylobacter coli are important zoonotic pathogens that are recognised as the principal causative agents of bacterial gastroenteritis [1, 2]. C. jejuni and C. coli are common commensals of poultry [3] with broiler chickens being the primary reservoir accounting for up to $80 \%$ of human infection [4]. These organisms are also common inhabitants of the gastrointestinal tract of other food producing animals such as cattle, pigs and sheep [5]. Dominant Campylobacter genotypes, belonging to the ST-21 clonal complex, ST-45 clonal complex and ST-828 clonal complex, exhibit a multi-host, generalist lifestyle [6-8]. By contrast, other $C$. jejuni lineages exhibit a host-adapted population structure in which certain genotypes are associated with a particular host species or ecological niche [9]. Similarly, certain lineages of $C$. coli have been linked to the swine production environment as well as the non-agricultural, environmental niche [10].

C. jejuni and C. coli show significant phenotypic diversity [11-15], and vary considerably in their ability to both adhere to and invade human intestinal epithelial cells in vitro [15]. Furthermore, C. jejuni genotypes vary in their infection ecology of the chicken host [16]. C. jejuni and $C$. coli show high mutation rates and are known to recombine with DNA obtained by natural transformation [17], a trait that drives population heterogeneity and can impact upon pathogenicity. For example, single nucleotide polymorphisms in porA, encoding the major outer membrane protein, have been shown to give rise to hyper-virulence in ruminants [18]. Many key surface molecules of Campylobacter are phase variable which may also impact upon variation in infection [19-22]. Large scale recombination within the Campylobacter genome, often associated with niche adaption has also been observed to impact upon infection potential [23].

Autotransporter proteins are the largest and most diverse class of secretory virulence determinants in Gramnegative bacteria [24, 25]. These surface-exposed or secreted proteins share a mechanism of export, conferred by their $C$-terminal $\beta$-barrel structure whilst virulence properties are conferred by their $\mathrm{N}$-terminal functional or "passenger" domain [24]. We recently described the CapC autotransporter in the commonly utilised reference strains C. jejuni 81,116 [26] and C. jejuni M1 [27], which is absent in the reference isolates $C$. jejuni NCTC 11168 and C. jejuni 81-176 [28]. Advances in sequencing technology have resulted in the public availability of large collections of genome sequences of $C$. jejuni and C. coli [29], which have been used to show distinct distribution patterns of gene families involved in pathogenesis, metabolism and stress responses [23, 30-32]. Autotransporter proteins often occur in families within a bacterial species or genus [33], and the distribution of such autotransporter families in isolates from distinct backgrounds may aid our understanding of phenotypic variation in Campylobacter species, and shed light on host specificity and niche adaption of different Campylobacter genotypes.

In this study we used publicly available Campylobacter genome sequences to demonstrate that the CapC autotransporter of $C$. jejuni 81,116 is a representative of a larger family of Campylobacter autotransporters. Furthermore, we identify a related family of autotransporters, CapD, that are related to, but distinct from $\mathrm{CapC}$, and have determined the distribution, genotype associations and extent of gene decay of the $\operatorname{cap} C$ and capD genes within the genus Campylobacter, focusing on C. jejuni and C. coli.

\section{Results}

\section{Identification of the capC and capD autotransporter} families in Campylobacter species

Initial screenings with the CapC protein sequence from C. jejuni 81,116 (C8J_1278) against C. jejuni and C. coli genomes from Genbank showed that there were several sequence variants present in addition to CapC in the $C$. jejuni and $C$. coli genome sequences. These autotransporter genes exhibited considerable sequence divergence in the N-terminal "passenger" domain yet share significant identity in their C-terminal domains (Fig. 1a) $[25,28]$. The phylogenetic tree in Fig. 1b shows that the newly identified CapC-like autotransporters separate into two, defined clusters; one which we named CapC as it includes the originally described $\operatorname{cap} C$ autotransporter described in C. jejuni 81,116 and C. jejuni M1 [28], designated capC1. Another cluster was named CapD and this encompasses the capD autotransporter family. In addition to the divergence in protein sequence, a major difference between the $c a p C$ and $c a p D$ autotransporter families is the location of a homopolymeric G-tract. In capC autotransporters, the poly- $\mathrm{G}$ tract is located upstream of the coding sequence in the putative promoter region whilst in the capD autotransporter family the poly-G tract is located in the coding sequence or is absent entirely (Fig. 1). Autotransporter genes belonging to the $\operatorname{cap} C$ family were identified in $C$. peloridis, $C$. ornithicola, C. lari, C. upsaliensis, C. subantarcticus and C. cuniculorum (Fig. 1c). Autotransporter genes belonging to the capD family were detected in C. ornithicola, C. volucris and C. subantarcticus (Fig. 1c). Alignment of the complete amino acid sequences of those autotransporters as well as alignment of only the C-terminal region of each autotransporter (Fig. 1c) illustrates the division of all autotransporters detected in Campylobacter into the distinct capC and capD families. The position of the poly-G tract for capC and $c a p D$ is conserved throughout the genus Campylobacter (Fig. 1c). 


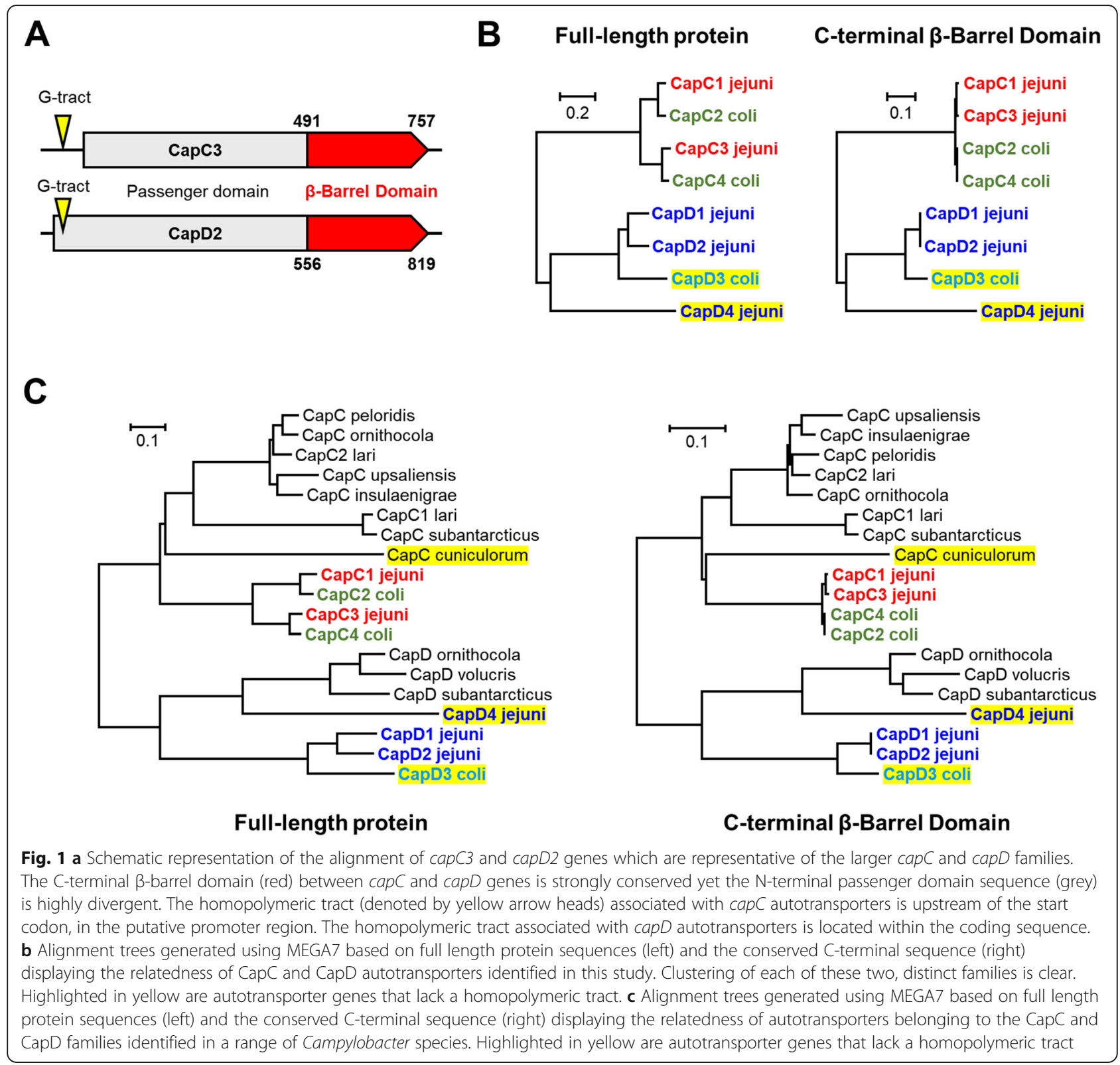

\section{Genetic characterisation of capC and capD}

\section{autotransporters in $C$. jejuni and $C$. coli}

In order to fully characterise the extent and distribution of autotransporter genes in $C$. jejuni and $C$. coli, each capC and capD variant was used to screen a collection of 5829 C. jejuni and 1347 C. coli genomes (Additional file 1). The $\operatorname{cap} C$ and $\operatorname{capD}$ autotransporters share a degree of similarity (Fig. 1a, b, Additional file 2) in their signal peptide and C-terminal $\beta$-barrel domain, but are highly dissimilar in the N-terminal domain. Genes belonging to the CapC family were tentatively designated capC2, capC3 and capC4, respectively, in addition to the original capC1 gene from $C$. jejuni 81,116. A high degree of sequence similarity was observed between capC1 and
capC2, and capC3 and capC4 (Fig. 1b). Genes belonging to the CapD family were designated as capD1, capD2 and capD4 in C. jejuni, and capD3 in C. coli. In C. jejuni and $C$. coli, the capC1-C4 genes were all present at the same genomic position, in between the ppk gene (encoding a polyphosphate kinase) and the $s s r A$ gene encoding a transfer-messenger RNA. These $\operatorname{cap} C$ genes are mutually exclusive as they occupy the same genomic position, suggesting recombination and genotype compatibility as the major driver of heterogeneity. We did not detect any genomes containing multiple $\operatorname{cap} C$ genes in their intact forms. The extended regions upstream and downstream of the $\operatorname{cap} C$ locus were largely conserved between strains except for the $c j 1365$ c gene in capC-negative strains. 
The capD1 and capD2 genes are also mutually exclusive in $C$. jejuni and $C$. coli and are present between the murA gene, involved in peptidoglycan synthesis and $f_{s p A 2}$, encoding a flagella-related protein [34]. This location is not conserved in $C$. coli Clade 3 which encodes the capD3 gene between the moeA gene, involved in molybdenum metabolism [35], and a tRNA/ATPase gene. In the single genome containing capD4, the gene is next to an $\mathrm{ABC}$ transporter encoding gene and a contig end.

As the N-terminal part of autotransporters often determines specific targets or functionality, we used predictive software algorithms to investigate the $\mathrm{CapC1}-\mathrm{C} 4$ and CapD1-D4 proteins. Autotransporter proteins display similarities and differences in their signal peptides, protein size and localisation (Additional file 5), which justifies their differentiation into separate families. CapC proteins have identical signal peptides and similar predicted protein sizes. However, CapC2 and CapC4 are predicted to have dual localisation sites in the outer membrane and secreted extracellularly. CapD autotransporters vary in their signal peptide composition and cleavage site as well as protein size. CapD1 and CapD2 are predicted to be secreted extracellularly, whereas CapD3 and CapD4 are predicted to localise to the outer membrane proteins. This indicates a high degree of structural conservation within the C-terminal of CapC and CapD autotransporter proteins, and a high degree of variation in the $\mathrm{N}$-terminal domains, but does not provide further information on functionality of these domains.

\section{Lineage-specific associations of intact and inactive autotransporters}

The 7176 C. jejuni and C. coli genome sequences (Additional file 1) were screened for the presence of $\operatorname{capC}$ and capD genes to determine whether the genes detected are intact and therefore predicted to encode a full-length protein, or whether the genes detected are inactive and predicted not to encode a functional protein (Figs. 2 and 3, Table 1, Table 2, Additional file 1). Autotransporter genes, in both intact and inactive formats, are present in most clonal complexes in $C$. jejuni and $C$. coli although there were notable associations with specific C. jejuni and C. coli genetic backgrounds. For example, whilst there are instances of capC1 in genomes belonging to numerous clonal complexes, it is predominantly associated with the ST-283 clonal complex and a sub-group of the ST-45 clonal complex (Fig. 2). Moreover, the distribution of intact and inactive autotransporter genes was associated with specific MLST genotypes of $C$. jejuni and C. coli. For instance, inactive capC3 is highly pervasive in $C$. jejuni and is present in a wide range of MLST genotypes including the ST-658, ST-52, ST-574, ST-354, ST-443, ST-353, ST-464, ST-573 ST61, ST-206 and ST-48 clonal complexes. However, the complete, intact gene is mostly present in the ST-45 clonal complex and the ST-573 clonal complex. Similarly, the capC4 gene is associated with numerous clonal complexes in its complete, intact form, but is inactive in the ST-257 clonal complex (Fig. 2, Additional file 1). This apparent linkage of inactive and intact autotransporter genes with genetic background is also observed in C. coli which has a more defined genomic population structure. The capC1-C4 autotransporters are closely associated with $C$. coli Clade1a/ST-828 and are absent from Clade 2 and 3, whereas the capD3 autotransporter is exclusively associated with C. coli Clade 3 .

\section{Homopolymeric G-tract length does not influence intact or inactive status of $c a p C$}

Homopolymeric guanine/cytosine tracts mediate adaptive mutations in Campylobacter species through slipped-strand mispairing of these repetitive sequences [21, 36]. Variation in the homopolymeric tract identified in the coding sequence of capD autotransporters will influence inactivation of capD genes, but whether the poly-G tract upstream of capC genes influences inactivation of the downstream gene was not known. The poly-G tract upstream of the capC1 start codon in the $C$. jejuni 81,116 reference genome is also present at the equivalent site in capC-C4-positive genomes (Fig. 1a). To determine whether this homopolymeric tract influenced the observed inactivation of $\operatorname{cap} C$ genes, we compared the length of poly-G tracts with the active/inactive status of the downstream autotransporter gene (Fig. 2 and Fig. 3). In $C$. jejuni, tract length ranged from $G=4$ to $G \geq 10$ and the most common tract length was G9 (Fig. 2, Additional file 1). $\operatorname{cap} C$ autotransporters within the same clonal complex were determined to be intact at a range of poly-G tract lengths; for example, in ST-45 the complete, intact capC1 and capC3 are present with poly-G tract lengths of G4 to G10. Similarly, the Gtract length of inactive capC4 in C. jejuni ST-257 ranges from $G 8$ to $G \geq 10$. Furthermore, in C. coli, intact and inactive $\operatorname{cap} C$ autotransporters were present with tract lengths of G7, G8, G9 and G10. These results indicate that homopolymeric tract length does not correspond with whether $\operatorname{capC}$ autotransporter genes are intact or inactive and that intact or inactive status of $\operatorname{capC}$ autotransporters is closely associated with clonal complex (Additional files 3 and 4).

\section{Discussion}

The autotransporter family is comprised of many important bacterial virulence factors in Gram-negative pathogens [24, 33]. These proteins consist of an N-terminal "passenger" domain which determines the effector function of the autotransporter [24], and a C-terminal $\beta$-barrel domain which facilitates insertion into the bacterial outer-membrane [25]. The CapC1 autotransporter has been shown to contribute 


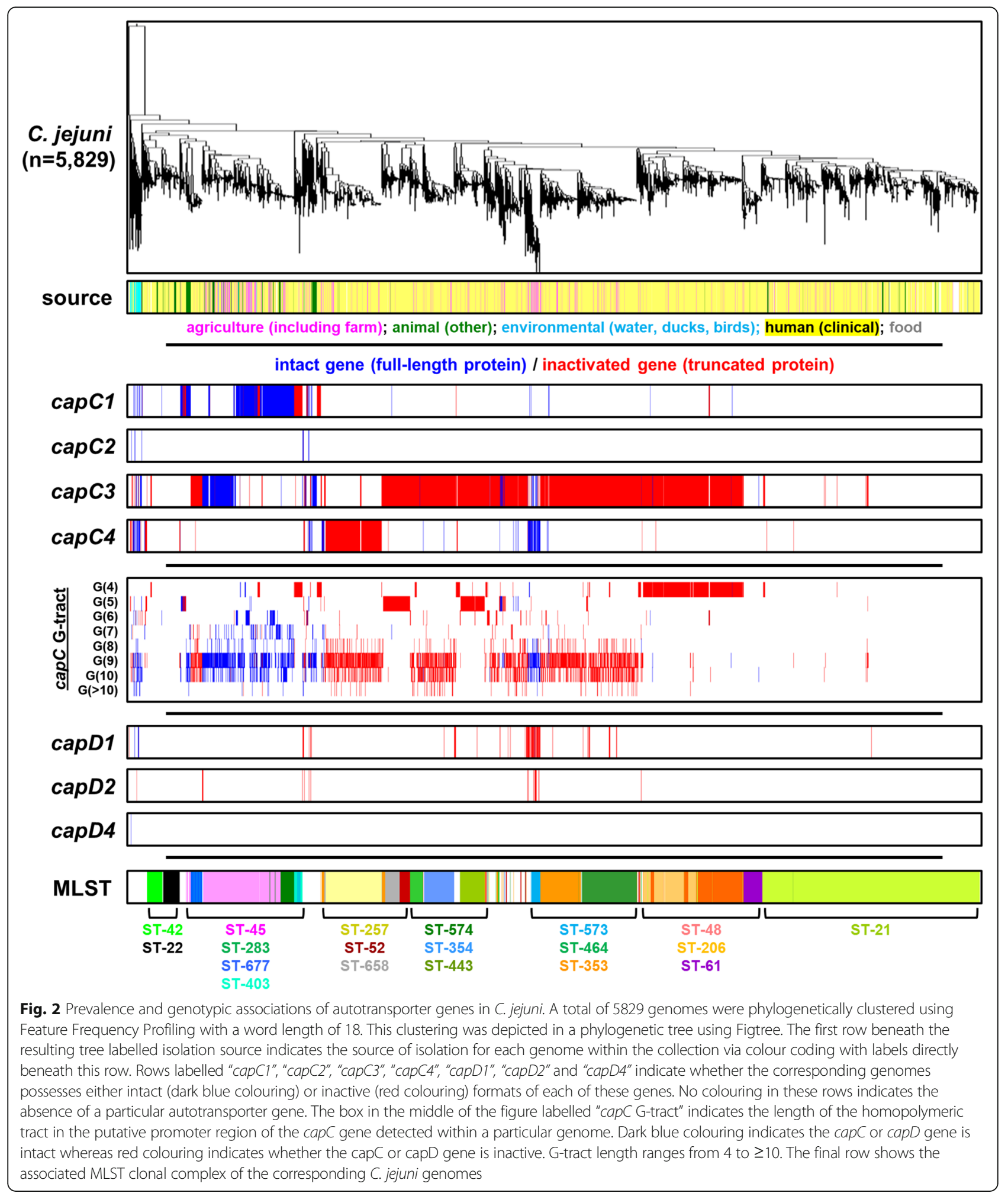

to virulence in $C$. jejuni and the CapA autotransporter has been reported to be involved in adhesion to epithelial cells and chicken colonisation [28, 37, 38], although we do not yet know the exact mechanism by which CapC1 contributes to virulence. Bioinformatic analysis of the passenger domains of CapC1-C4 and CapD1-D4 did not result in identification of specific domains that may explain such functionality (Additional file 5). 


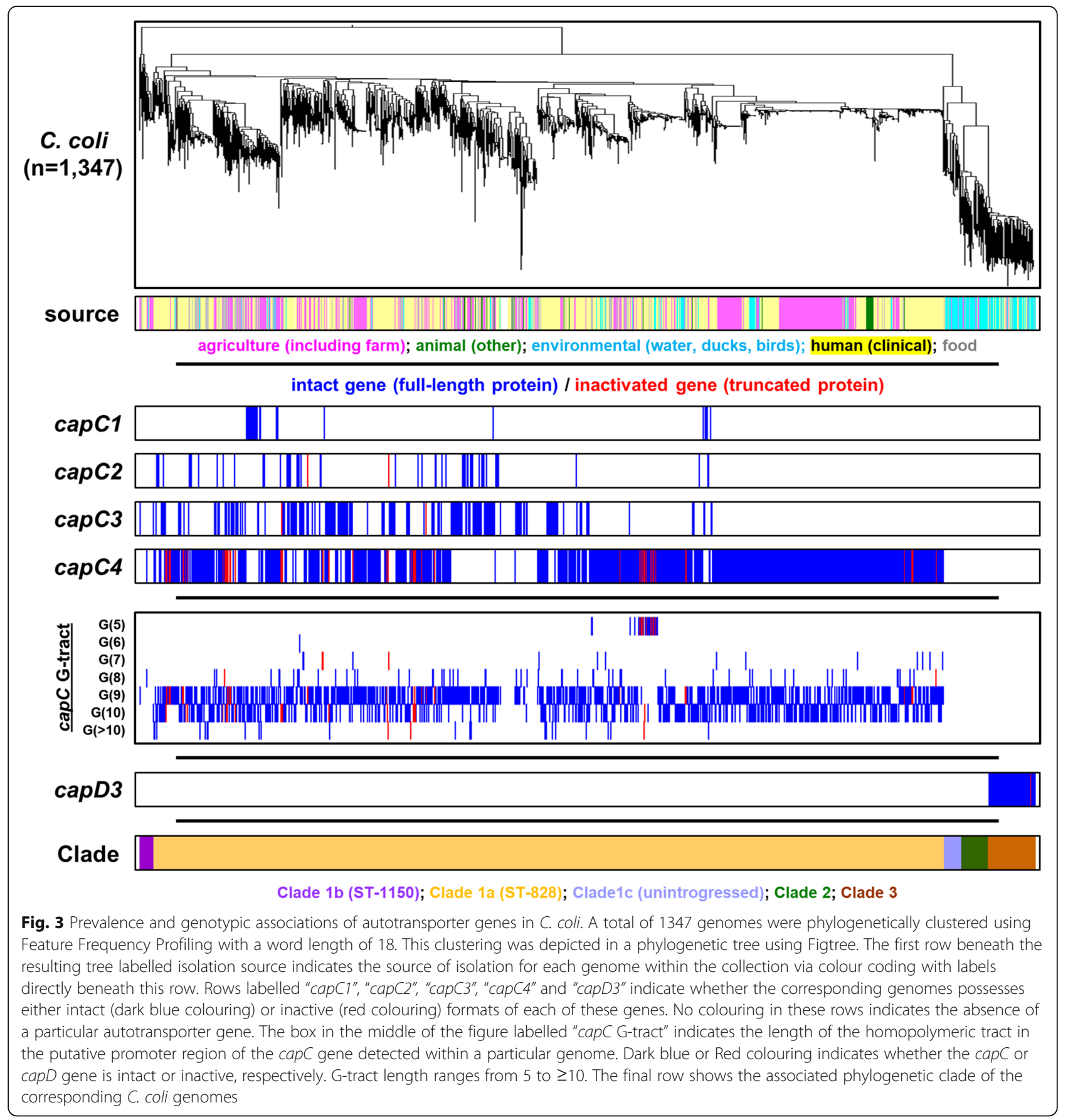

In this study, we have described two novel autotransporter families in Campylobacter and report the lineage-specific distribution and decay of these autotransporter genes. Notably, we determined that $\operatorname{cap} C$ autotransporters are shared between $C$. jejuni and $C$. coli lineages [39]. The capC and capD autotransporter genes are common throughout $C$. jejuni and C. coli in either their inactive or intact forms, except for select lineages which do not appear to encode CapC- or CapD autotransporters (Additional file 1). There is a clear, defined sub-population within ST-45 containing capC3 rather than capC1. The degree of demarcation between lineages that encode certain autotransporters is exemplified by this sub-population and is evidence of strong genotype associations rather than with isolation source. Due the linkage of genotype and ecological niche observed in Campylobacter [9], observed associations of an autotransporter with a particular genetic lineage may cause an indirect association with an isolation source. These associations may be exaggerated considering that the 
Table 1 The number and proportion of genomes within major C. jejuni clonal complexes and C. coli Clades from the collection used in this study that encode intact and inactive capC autotransporter genes. The number and proportion of genomes that do not encode capC or capD is also shown

\begin{tabular}{|c|c|c|c|c|c|c|c|c|c|c|c|c|c|c|c|c|c|c|c|}
\hline \multirow{3}{*}{$\begin{array}{l}\text { Clonal } \\
\text { Complex } \\
\text { ST-21 }\end{array}$} & \multirow{3}{*}{$\begin{array}{l}\text { Total } \\
\text { Genomes } \\
\mathbf{1 5 0 0}\end{array}$} & \multicolumn{4}{|c|}{ capC1 } & \multicolumn{4}{|c|}{ capC2 } & \multicolumn{4}{|c|}{$\operatorname{capC3}$} & \multicolumn{4}{|c|}{$\operatorname{capC4}$} & \multirow{2}{*}{\multicolumn{2}{|c|}{$\frac{\boldsymbol{c a p C} / \mathbf{c a p D}}{\text { absent }}$}} \\
\hline & & \multicolumn{2}{|c|}{ Intact } & \multicolumn{2}{|c|}{ Inactive } & \multicolumn{2}{|c|}{ Intact } & \multicolumn{2}{|c|}{ Inactive } & \multicolumn{2}{|c|}{ Intact } & \multicolumn{2}{|c|}{ Inactive } & \multicolumn{2}{|c|}{ Intact } & \multicolumn{2}{|c|}{ Inactive } & & \\
\hline & & 0 & - & 0 & - & 0 & - & 0 & - & 0 & - & 45 & $(3 \%)$ & 0 & - & 2 & $(0.13 \%)$ & 1452 & $(96.8 \%)$ \\
\hline ST-22 & 112 & 0 & - & 0 & - & 0 & - & 0 & - & 0 & - & 0 & - & 0 & - & 0 & - & 112 & (100\%) \\
\hline ST-42 & 105 & 1 & $(0.95 \%)$ & 0 & - & 0 & - & 0 & - & 0 & - & 8 & (7.61\%) & 0 & - & 0 & - & 96 & (91.4\%) \\
\hline ST-45 & 543 & 309 & (56.9\%) & 20 & $(3.68 \%)$ & 0 & - & 0 & - & 203 & (37.3\%) & 9 & $(1.65 \%)$ & 0 & - & 0 & - & 2 & $(0.36 \%)$ \\
\hline ST-48 & 375 & 2 & $(0.53 \%)$ & 7 & $(1.86 \%)$ & 0 & - & 0 & - & 3 & $(0.8 \%)$ & 361 & $(96.2 \%)$ & 0 & - & 0 & - & 2 & $(0.53 \%)$ \\
\hline ST-52 & 82 & 0 & - & 0 & - & 0 & - & 0 & - & 0 & - & 82 & (100\%) & 0 & - & 0 & - & 0 & - \\
\hline ST-61 & 130 & 0 & - & 0 & - & 0 & - & 0 & - & 1 & $(0.76 \%)$ & 4 & (3.07\%) & 0 & - & 0 & - & 125 & (96.1\%) \\
\hline ST-206 & 300 & 0 & - & 0 & - & 0 & - & 0 & - & 0 & - & 297 & (99\%) & 0 & - & 1 & $(0.33 \%)$ & 2 & $(0.66 \%)$ \\
\hline ST-257 & 394 & 0 & - & 0 & - & 0 & - & 0 & - & 0 & - & 19 & $(4.82 \%)$ & 0 & - & 375 & (95.1\%) & 0 & - \\
\hline ST-283 & 99 & 98 & (98.9\%) & 0 & - & 0 & - & 0 & - & 1 & $(1.01 \%)$ & 0 & - & 0 & - & 0 & - & 0 & - \\
\hline ST-353 & 339 & 4 & $(1.17 \%)$ & 0 & - & 0 & - & 0 & - & 3 & $(0.88 \%)$ & 311 & $(91.7 \%)$ & 18 & $(5.30 \%)$ & 1 & $(0.29 \%)$ & 2 & $(0.58 \%)$ \\
\hline ST-354 & 214 & 0 & - & 0 & - & 0 & - & 0 & - & 0 & - & 213 & (99.5\%) & 1 & $(0.46 \%)$ & 0 & - & 0 & - \\
\hline ST-403 & 56 & 0 & - & 55 & $(98.2 \%)$ & 0 & - & 0 & - & 0 & - & 1 & $(1.78 \%)$ & 0 & - & 0 & - & 0 & - \\
\hline ST-443 & 168 & 0 & - & 0 & - & 0 & - & 0 & - & 1 & $(0.59 \%)$ & 0 & - & 3 & (1.78\%) & 0 & - & 0 & - \\
\hline ST-464 & 379 & 0 & - & 0 & - & 0 & - & 0 & - & 0 & - & 377 & (99.4\%) & 0 & - & 0 & - & 2 & $(0.52 \%)$ \\
\hline ST-573 & 61 & 1 & $(1.63 \%)$ & 0 & - & 0 & - & 0 & - & 14 & $(22.9 \%)$ & 3 & (4.91\%) & 43 & $(70.4 \%)$ & 0 & - & 0 & - \\
\hline ST-574 & 99 & 0 & - & 0 & - & 0 & - & 0 & - & 3 & (3.03\%) & 96 & (96.9\%) & 0 & - & 0 & - & 0 & - \\
\hline ST-658 & 110 & 1 & $(0.90 \%)$ & 0 & - & 0 & - & 0 & - & 0 & - & 108 & (98.1\%) & 0 & - & 1 & $(0.90 \%)$ & 0 & - \\
\hline ST-677 & 78 & 0 & - & 0 & - & 0 & - & 0 & - & 0 & - & 77 & $(98.7 \%)$ & 0 & - & 1 & $(1.28 \%)$ & 0 & - \\
\hline None & 434 & 26 & (5.99\%) & 5 & $(1.15 \%)$ & 10 & $(2.30 \%)$ & 1 & $(0.23 \%)$ & 65 & (14.9\%) & 222 & $(51.1 \%)$ & 32 & $(7.37 \%)$ & 25 & $(5.76 \%)$ & 46 & $(10.5 \%)$ \\
\hline $\begin{array}{l}\text { Clade1a } \\
\text { (ST-828) }\end{array}$ & 1189 & 29 & (2.43\%) & 0 & - & 60 & (5.04\%) & 2 & (0.16\%) & 204 & (17.1\%) & 3 & $(0.25 \%)$ & 787 & $(66.1 \%)$ & 51 & $(4.28 \%)$ & 54 & (4.54\%) \\
\hline $\begin{array}{l}\text { Clade1b } \\
\text { (ST-1150) }\end{array}$ & 20 & 0 & - & 0 & - & 0 & - & 0 & - & 1 & $(5 \%)$ & 0 & - & 1 & $(5 \%)$ & 0 & - & 18 & $(90 \%)$ \\
\hline Clade1c & 26 & 0 & - & 0 & - & 0 & - & 0 & - & 0 & - & 0 & - & 0 & - & 0 & - & 26 & $(100 \%)$ \\
\hline Clade 2 & 40 & 0 & - & 0 & - & 0 & - & 0 & - & 0 & - & 0 & - & 0 & - & 0 & - & 40 & (100\%) \\
\hline Clade 3 & 72 & 0 & - & 0 & - & 0 & - & 0 & - & 0 & - & 0 & - & 0 & - & 0 & - & 3 & (4.16\%) \\
\hline
\end{tabular}

Table 2 The number and proportion of genomes within major C. jejuni clonal complexes and C. coli Clades from the collection used in this study that encode intact and inactive capD autotransporter genes

\begin{tabular}{|c|c|c|c|c|c|c|c|c|c|c|c|c|c|c|c|c|c|}
\hline \multirow{3}{*}{$\begin{array}{l}\text { Clonal } \\
\text { Complex } \\
\text { ST-353 }\end{array}$} & \multirow{3}{*}{$\begin{array}{l}\text { Total } \\
\text { Genomes } \\
\mathbf{3 3 9}\end{array}$} & \multicolumn{4}{|c|}{ capD1 } & \multicolumn{4}{|c|}{ capD2 } & \multicolumn{4}{|c|}{ capD3 } & \multicolumn{4}{|c|}{ capD4 } \\
\hline & & \multicolumn{2}{|c|}{ Intact } & \multicolumn{2}{|c|}{ Inactive } & \multicolumn{2}{|c|}{ Intact } & \multicolumn{2}{|c|}{ Inactive } & \multicolumn{2}{|c|}{ Intact } & \multicolumn{2}{|c|}{ Inactive } & \multicolumn{2}{|c|}{ Intact } & \multicolumn{2}{|c|}{ Inactive } \\
\hline & & 0 & - & 1 & $(0.29 \%)$ & 0 & - & 0 & - & & & & & 0 & - & 0 & - \\
\hline ST-354 & 214 & 0 & - & 2 & (0.93\%) & 0 & - & 0 & - & & & & & 0 & - & 0 & - \\
\hline ST-443 & 168 & 0 & - & 1 & (0.59\%) & 0 & - & 0 & - & & & & & 0 & - & 0 & - \\
\hline ST-464 & 379 & 0 & - & 21 & (5.54\%) & 0 & - & 0 & - & & & & & 0 & - & 0 & - \\
\hline ST-573 & 61 & 1 & $(1.63 \%)$ & 54 & (88.50\%) & 0 & - & 13 & (21.30\%) & & & & & 0 & - & 0 & - \\
\hline ST-661 & 13 & 0 & - & 10 & (76.90\%) & 0 & - & 1 & (7.69\%) & & & & & 0 & - & 0 & - \\
\hline ST-692 & 12 & 0 & - & 1 & (8.33\%) & 0 & - & 0 & - & & & & & 0 & - & 0 & - \\
\hline None & 434 & 4 & $(0.92 \%)$ & 47 & (10.80\%) & 0 & - & 14 & (3.22\%) & & & & & 1 & $(0.23 \%)$ & 0 & - \\
\hline Clade 3 & 72 & & & & & & & & & 68 & (94.40\%) & 1 & (1.38\%) & & & & \\
\hline
\end{tabular}


collection of publicly available Campylobacter genomes used in this study is heavily comprised of human clinical isolates belonging to ST-21 and Clade 1a C. coli, which are more readily available than isolates from other sources. Human infections are commonly transmitted via poultry or ruminant sources, but for these human isolates the transmission route is not known. The high frequency with which ST-21 and Clade 1a C. coli isolates appear in the dataset can skew interpretations regarding the proportion of autotransporter genes encoded by Campylobacter. Ecological association displayed by certain genotypes does not preclude events leading to transmission of isolates to different niches. Definitive source attribution is difficult in Campylobacter species [40, 41], particularly with multi-host adapted lineages which display poor host specificity markers [6]. Therefore, potential associations of autotransporters with ecological niches via quantitative source attribution, are difficult to accurately infer. Ultimately, possession of $\operatorname{cap} C$ and capD autotransporters is correlated with the genetic background of $C$. jejuni and C. coli.

Intact $\operatorname{cap} C$ autotransporters are predominantly associated with the ST-45 and ST-283 clonal complexes in C. jejuni and the ST-828 (Clade 1a) clonal complexes in C. coli. Considering the high degree of inactive capC genes in other clonal complexes, the high proportion of intact, functional capC in ST-45 and ST-828 is striking and could be indicative of a functional role for these autotransporters in colonisation of the agricultural niche or in the multi-host lifestyle exhibited by these lineages. However, ST-21 is also a generalist lineage that is prevalent within the agricultural niche, yet isolates from this clonal complex do not contain the $\operatorname{capC}$ autotransporter gene whilst thriving in these environments [42]. Rather, $C$. jejuni ST-21 often contains the cap $A / B$ autotransporter genes, which may mitigate for the absence of CapC or CapD autotransporters [28, 37]. Previous studies have demonstrated that $C$. jejuni isolates from generalist lineages readily recombine with each other in vitro, yet despite a considerable degree of niche overlap, the ST-45 and ST-21 lineages do not show any evidence of recombination with each other in nature [7]. Therefore, the ecological barrier that segregates these lineages may also restrict capC autotransporter genes to ST-45 and ST-828.

We have identified shared capC1-C4 autotransporter genes between $C$. jejuni lineages and introgressed C. coli ST-828 (Figs. 2, 3). In C. jejuni, the CapC autotransporter family is restricted to select genotypes in either its intact or inactive form. However, C. coli ST-828 encodes predominantly intact capC1-C4 autotransporters with no discernible association of each $\operatorname{cap} C$ allele with subpopulation structure of ST-828. Considering the similarity between $\operatorname{capC}$ autotransporter genes (Fig. 1) as well as the upstream and downstream genes, this observed incidence is consistent with interspecies sharing of capC autotransporters between $C$. coli Clade1a/ST-828 and multiple $C$. jejuni lineages, probably via a shared niche. Recombination between $C$. jejuni and C. coli ST-828 has been demonstrated previously by the accumulation of $C$. jejuni alleles by C. coli $[39,43,44]$.

Both capC and capD have homopolymeric G-tracts associated with the genes, but their respective position is distinct. The $\operatorname{capC}$ genes have poly-G tract upstream of the $\operatorname{cap} C$ start codon, whereas the $\operatorname{capD}$ genes have a poly-G tract in the open reading frame or do not have a poly-G tract at all. Our analysis shows that the length of this poly-G tract, whilst variable, does not correlate with the inactive/intact status of the $\operatorname{cap} C$ autotransporters and therefore does not influence inactivation of these genes (Figs. 2, 3). Coupled with the association of intact and inactivated formats with specific clonal complexes, we propose that inactivation of these genes is linked with Campylobacter genotype rather than homopolymeric tract length. Exceptions to this are the inactive capC3 genes in the ST-48, ST-206 and ST-61 clonal complexes which predominantly possess a G-tract of 4 consecutive nucleotides and those in ST-443, ST-52 and ST-658 which possess G-tracts of 5 nucleotides. The capC3 gene in these lineages all display highly similar patterns of inactivation (Additional files 3 and 4) and are decayed to such an extent as to make reversion to intact status by addition or deletion of a nucleotides upstream of the coding sequence impossible. The uniform G-tract length in these clonal complexes is likely the result of gene decay of the entire locus including the intergenic regions due to lack of maintenance pressure. It is therefore likely that a progressive process of pseudogene formation is responsible for degradation of autotransporter genes in specific lineages rather than phase variation mediated by poly-G tracts. Pseudogenisation of autotransporters suggests a functional redundancy of these genes in certain lineages, leading to inactivation once their respective functions are no longer required within a specialised niche $[45,46]$. This "adaptive loss" scenario has been observed in C. jejuni previously and is a proposed consequence of niche differentiation [45]. Conversely, this would suggest a possible environmental pressure selecting for the maintenance of intact capC and capD in C. jejuni ST-45 and ST-283 and C. coli ST-828 and for capD3 in C. coli Clade 3. Given the location of the poly-G tract, it is conceivable that strand-slippage may impact upon the expression of the capC genes [26, 28]. Furthermore, given the widespread sharing of capC autotransporters, it is possible that the intergenic regions upstream and downstream are also shared by interlineage and inter-species recombination making evaluation of the impact of homopolymeric tract length very difficult. 


\section{Conclusions}

In this study we report on two novel, related autotransporter families in the genus Campylobacter and show that $\operatorname{cap} C$ and $c a p D$ autotransporter genes display specific distribution patterns of intact and inactive genes associated with MLST clonal complexes. This widespread, lineage-specific inactivation of $\operatorname{cap} C$ and $\operatorname{capD}$ genes in Campylobacter likely represents gene decay as a consequence of functional redundancy, host/niche adaption or a lack of environmental selection towards maintenance of intact genes, especially in C. jejuni. The select presence of autotransporters highlights that Campylobacter virulence mechanisms vary between strains and genetic backgrounds and that accessory gene distribution and decay is an important consideration when evaluating Campylobacter phenotypic variability. This contrasts with capC genes being exchanged between $C$. jejuni and $C$. coli, presumably via a shared environment and recombination. Furthermore, this pattern of genetic exchange highlights the erosion of intrinsic recombination barriers between these species arising through co-habitation. Further studies are required to fully examine interspecies recombination of capC autotransporters, and whether the barriers that prevent recombination of these autotransporters, thus restricting them to certain lineages, are ecological or essential in nature. Other virulence determinants and metabolic genes should also be examined to more accurately define genotype associations and the extent of exchange of genetic material between $C$. jejuni and $C$. coli.

\section{Methods}

\section{Campylobacter genomes used in this study}

A collection of 7176 complete and draft Campylobacter genome sequences (5829 C. jejuni, 1347 C. coli) were used in this study and obtained from PubMLST (http:// pubmlst.org/campylobacter) and Genbank. These genomes are listed in Additional file 1, with PubMLST ID, Genbank accession number, isolate source category, MLST sequence type, clonal complex, capC1-4 and capD1-4 status and G-tract length capC genes included where available. For $C$. coli, the clades 1a (ST-828), clade 1b (ST-1150), clade 1c (non-introgressed), clade 2 and clade $3[30,39]$ were also determined. The assembly quality of genome assemblies was evaluated using Quast V 4.6.3 [47] and poor quality assemblies were excluded, based on aberrant genome size $(<1.5 \mathrm{Mbp}$ or $>2.0$ $\mathrm{Mbp})$, low $\mathrm{N}_{50}(<25 \mathrm{kbp})$, high $\mathrm{L}_{50}(>25)$, and high number of Ns per $100 \mathrm{~kb}(>50)$.

\section{Determination of the prevalence of intact and inactive autotransporters in $C$. jejuni and $C$. coli}

Genome sequences were screened for the presence of the capC1-4 and capD1-4 genes by using Abricate version 0.8 (https://github.com/tseemann/abricate) and
BLAST+ version 2.9.0 (NCBI). All genomes in the collection were annotated using Prokka [48], and these annotations were screened for complete and truncated versions of the CapC1-4 and CapD1-4 proteins using BioEdit version 7.25 [49]. The G-tract length of the capC1-4 promoters was determined after querying the genome sequences with the promoter of the capC1 gene of C. jejuni 81,116 (C8J_ 1278). Phylogenetic trees were created for $C$. jejuni and $C$. coli genomes using Feature Frequency Profiling with a word length of 18 [50], as used previously for earlier collections of $C$. jejuni and $C$. coli genome sequences [31, 32]. Colour-coding of intact and inactive gene encoding isolates within a phylogenetic tree generated using Figtree allowed associations of autotransporters with genotypes to be visualised.

\section{Bioinformatic tools for comparison of CapC and CapD autotransporter families}

SignalP 5.0 (http://www.cbs.dtu.dk/services/SignalP/), CELLO V2.5 (http://cello.life.nctu.edu.tw/), NCBI Conserved Domain Database (http://www.ncbi.nlm.nih.gov/ Structure/cdd/wrpsb.cgi), Phyre2 (http://www.sbg.bio.ic.ac. uk/phyre2/html/page.cgi?id=index) and Protein Molecular Weight Calculator (https://www.bioinformatics.org/sms/ prot_mw.html) were used to identify signal peptides, conserved domains, autotransporter protein size and subcellular localisation of CapC and CapD autotransporters.

\section{Supplementary information}

Supplementary information accompanies this paper at https://doi.org/10. 1186/s12864-020-6704-z.

Additional file 1. Table of C. jejuni and C. coli genomes used in this study showing Genbank accession numbers, capC/capD presence or absence and intact or inactivation status.

Additional file 2. A) Alignment of amino acid sequences of CapC1/2/3/ 4 and CapD1/2/3/4 variants in C. jejuni and C. coli. B) Alignment of amino acid sequence of CapC and CapD variants in Campylobacter species.

Additional file 3. Figure displaying the fragmentation patterns of inactive capC3 and capC4 genes. The figure shows the various frameshifts (FS) and point mutations that result in inactive genes; these mutations are associated with the clonal complex in which the inactive capC3/ capC4 is present.

Additional file 4. A) Table showing the number of genomes in major clonal complexes that encode frameshift (FS) or premature stop mutations in CapC3/CapC4 at specific amino acid residues. B) Table showing the percentage of genomes in major clonal complexes that encode frameshift (FS) or premature stop mutations in CapC3/CapC4 at specific amino acid residues.

Additional file 5. Table showing summary of results from comparison of autotransporter amino acid sequences using searching for conserved domains, signal sequences, protein size and predicted localisation sites.

\section{Abbreviations}

C. jejuni: Campylobacter jejuni; C. coli: Campylobacter coli; ST: Sequence Type; MLST: Multi-Locus Sequence Type; tRNA: transfer-ribonucleic acid; ATPase: adenosine triphosphate hydrolase; Poly-G: homopolymeric guanine tract; bp: base pairs 


\section{Acknowledgements}

We wish to thank Dr. Simon Park for his advice and constructive discussion on the manuscript.

\section{Authors' contributions}

$J M, R L R$ and AvV conceived the study. AvV and JM performed the data collection. JM and AvV contributed to methodology and data analysis and interpretation. JM analysed and discussed data and wrote the paper. AvV and RLR were major contributors in writing the manuscript. All authors read and approved the final manuscript.

\section{Funding}

Not applicable/This work received no funding.

\section{Availability of data and materials}

All data generated or analysed during this study are included in this published article [and its Additional information files] and is publicly available from http://pubmlst.org/campylobacter and https://www.ncbi.nlm.nih.gov/ genome.

Genbank Accession Numbers: C. jejuni CapC1: WP_002866779.1; C. coli CapC2: WP_052793243.1; C. jejuni CapC3: WP_022552386.1; C. coli CapC4: WP_023362112.1; C. jejuni CapD1: WP_126232584.1; C. jejuni CapD2: WP_126216674.1; C. coli CapD3: WP_020974791.1; C. jejuni CapD4: WP_070298870.1; C. lari CapC1: WP_114640428.1; C. lari CapC2: WP_074691797.1; C. peloridis CapC: WP_044598937.1; C. ornithocola CapC: WP_066008681.1; C. insulaenigrae CapC: WP_039650305.1; C. cuniculorum CapC: ARJ56787.1; C. volucris CapD: WP_039665304.1; C. upsaliensis CapC: translated as frameshifted protein from NZ_UFUZ01000001.1; C. subantarcticus CapC: WP_039664182.1 (N-terminus) and WP_082018437.1 (Cterminus); C. ornithocola CapD: OCX42345.1 (C-terminal part, N-terminal part translated from genome sequence LXSU01000139.1); C. subantarcticus CapD: $\mathrm{N}$-terminus translated from genome sequence, MPB98625.1 (middle part), MPB98624.1 (C-terminus).

\section{Ethics approval and consent to participate}

Not applicable.

\section{Consent for publication}

Not applicable.

\section{Competing interests}

The authors declare that they have no competing interests.

Received: 13 December 2019 Accepted: 27 March 2020 Published online: 19 April 2020

\section{References}

1. Tam CC, O'Brien SJ, Tompkins DS, Bolton FJ, Berry L, Dodds J, et al. Changes in causes of acute gastroenteritis in the United Kingdom over 15 years: microbiologic findings from 2 prospective, population-based studies of infectious intestinal disease. Clin Infect Dis. 2012;54:1275-86. https://doi.org/ 10.1093/cid/cis028.

2. Burnham PM, Hendrixson DR. Campylobacter jejuni: collective components promoting a successful enteric lifestyle. Nat Rev Microbiol. 2018;:1. doi: https://doi.org/10.1038/s41579-018-0037-9.

3. Hofreuter D. Defining the metabolic requirements for the growth and colonization capacity of Campylobacter jejuni. Front Cell Infect Microbiol. 2014:4:137. https://doi.org/10.3389/fcimb.2014.00137.

4. Gormley FJ, Bailey RA, Watson KA, McAdam J, Avendaño S, Stanley WA, et al. Campylobacter colonization and proliferation in the broiler chicken upon natural field challenge is not affected by the bird growth rate or breed. Appl Environ Microbiol. 2014;80:6733-8. https://doi.org/10.1128/AEM. 02162-14.

5. Manning G, Dowson CG, Bagnall MC, Ahmed IH, West M, Newell DG. Multilocus sequence typing for comparison of veterinary and human isolates of Campylobacter jejuni. Appl Environ Microbiol 2003;69:6370-6379. http://www.ncbi.nlm.nih.gov/pubmed/14602588. Accessed 21 June 2017.

6. Dearlove BL, Cody AJ, Pascoe B, Méric G, Wilson DJ, Sheppard SK. Rapid host switching in generalist Campylobacter strains erodes the signal for tracing human infections. ISME J. 2016;10:721-9. https://doi.org/10.1038/ ismej.2015.149.
7. Sheppard SK, Cheng L, Meric G, de Haan CPA, Llarena A-K, Marttinen P, et al. Cryptic ecology among host generalist Campylobacter jejuni in domestic animals. Mol Ecol. 2014;23:2442-51. https://doi.org/10.1111/ mec.12742.

8. Gripp E, Hlahla D, Didelot X, Kops F, Maurischat S, Tedin K, et al. Closely related Campylobacter jejuni strains from different sources reveal a generalist rather than a specialist lifestyle. BMC Genomics. 2011;12:584. https://doi.org/ 10.1186/1471-2164-12-584

9. Sheppard SK, Colles FM, McCarthy ND, Strachan NJC, Ogden ID, Forbes K, et al. Niche segregation and genetic structure of Campylobacter jejuni populations from wild and agricultural host species. Mol Ecol. 2011;20:348490. https://doi.org/10.1111/j.1365-294X.2011.05179.x.

10. Thakur S, Gebreyes WA. Campylobacter coli in swine production: antimicrobial resistance mechanisms and molecular epidemiology. J Clin Microbiol. 2005;43: 5705-14. https://doi.org/10.1128/JCM.43.11.5705-5714.2005.

11. O Cróinín T, Backert S. Host epithelial cell invasion by Campylobacter jejuni: trigger or zipper mechanism? Front Cell Infect Microbiol. 2012;2:25. doi: https://doi.org/10.3389/fcimb.2012.00025.

12. Anjum A, Brathwaite KJ, Aidley J, Connerton PL, Cummings NJ, Parkhill J, et al. Phase variation of a type $\| G$ restriction-modification enzyme alters site-specific methylation patterns and gene expression in Campylobacter jejuni strain NCTC11168. Nucleic Acids Res. 2016;44:4581-94. https://doi.org/ 10.1093/nar/gkw019.

13. Coward C, van Diemen PM, Conlan AJK, Gog JR, Stevens MP, Jones MA, et al. Competing isogenic Campylobacter strains exhibit variable population structures in vivo. Appl Environ Microbiol. 2008;74:3857-67. https://doi.org/ 10.1128/AEM.02835-07.

14. Bae W, Kaya KN, Hancock DD, Call DR, Park YH, Besser TE. Prevalence and antimicrobial resistance of thermophilic Campylobacter spp. from cattle farms in Washington state. Appl Environ Microbiol. 2005;71:169-74. https:// doi.org/10.1128/AEM.71.1.169-174.2005.

15. Fearnley C, Manning G, Bagnall M, Javed MA, Wassenaar TM, Newell DG Identification of hyperinvasive Campylobacter jejuni strains isolated from poultry and human clinical sources. J Med Microbiol. 2008;57:570-80. https://doi.org/10.1099/jmm.0.47803-0.

16. Chaloner G, Wigley P, Humphrey S, Kemmett K, Lacharme-Lora L, Humphrey $T$, et al. Dynamics of dual infection with Campylobacter jejuni strains in chickens reveals distinct strain-to-strain variation in infection ecology. Appl Environ Microbiol. 2014;80:6366-72. https://doi.org/10.1128/AEM.01901-14.

17. Sheppard SK, Maiden MCJ. The evolution of Campylobacter jejuni and Campylobacter coli. Cold Spring Harb Perspect Biol. 2015;7:a018119. https:// doi.org/10.1101/cshperspect.a018119.

18. Wu Z, Periaswamy B, Sahin O, Yaeger M, Plummer P, Zhai W, et al. Point mutations in the major outer membrane protein drive hypervirulence of a rapidly expanding clone of Campylobacter jejuni. Proc Natl Acad Sci U S A. 2016;113:10690-5. https://doi.org/10.1073/pnas.1605869113.

19. Guerry P, Szymanski CM, Prendergast MM, Hickey TE, Ewing CP, Pattarini DL, et al. Phase variation of Campylobacter jejuni 81-176 Lipooligosaccharide affects Ganglioside mimicry and invasiveness in vitro. Infect Immun. 2002;70: 787-93. https://doi.org/10.1128//Al.70.2.787-793.2002.

20. Kim J-S, Artymovich KA, Hall DF, Smith EJ, Fulton R, Bell J, et al. Passage of Campylobacter jejuni through the chicken reservoir or mice promotes phase variation in contingency genes Cj0045 and Cj0170 that strongly associates with colonization and disease in a mouse model. Microbiology. 2012;158 Pt_5:1304-16. https://doi.org/10.1099/mic.0.057158-0.

21. Bayliss CD, Bidmos FA, Anjum A, Manchev VT, Richards RL, Grossier J-P, et al. Phase variable genes of Campylobacter jejuni exhibit high mutation rates and specific mutational patterns but mutability is not the major determinant of population structure during host colonization. Nucleic Acids Res. 2012:40:5876-89. https://doi.org/10.1093/nar/gks246.

22. Holt JP, Grant AJ, Coward C, Maskell DJ, Quinlan JJ. Identification of Cj1051C as a major determinant for the restriction barrier of Campylobacter jejuni strain NCTC11168. Appl Environ Microbiol. 2012;78:7841-8. https://doi.org/ 10.1128/AEM.01799-12.

23. Baig A, McNally A, Dunn S, Paszkiewicz KH, Corander J, Manning G. Genetic import and phenotype specific alleles associated with hyper-invasion in Campylobacter jejuni. BMC Genomics. 2015;16:852. https://doi.org/10.1186/ s12864-015-2087-y.

24. Nishimura K, Tajima N, Yoon Y-H, Park S-Y, Tame JRH. Autotransporter passenger proteins: virulence factors with common structural themes. J Mol Med. 2010;88:451-8. https://doi.org/10.1007/s00109-010-0600-y. 
25. Leo JC, Grin I, Linke D. Type V secretion: mechanism(s) of autotransport through the bacterial outer membrane. Philos Trans R Soc Lond Ser B Biol Sci. 2012;367:1088-101. https://doi.org/10.1098/rstb.2011.0208.

26. Pearson BM, Gaskin DJH, Segers RPAM, Wells JM, Nuijten PJM, van Vliet AHM The complete genome sequence of Campylobacter jejuni strain 81116 (NCTC11828). J Bacteriol. 2007;189:8402-3. https://doi.org/10.1128/JB.01404-07.

27. Friis C, Wassenaar TM, Javed MA, Snipen L, Lagesen K, Hallin PF, et al. Genomic characterization of Campylobacter jejuni strain M1. PLoS One. 2010; 5:e12253. https://doi.org/10.1371/journal.pone.0012253.

28. Mehat JW, Park SF, van Vliet AHM, La Ragione RM. CapC, a novel autotransporter and virulence factor of Campylobacter jejuni. Appl Environ Microbiol. 2018;:AEM.01032-18. doi:https://doi.org/10.1128/AEM.01032-18.

29. Jolley KA, Bray JE, Maiden MCJ. Open-access bacterial population genomics: BIGSdb software, the PubMLST.org website and their applications. Wellcome Open Res. 2018;3:124. https://doi.org/10.12688/wellcomeopenres.14826.1.

30. Pearson BM, Louwen $R$, van Baarlen P, van Vliet AHM. Differential distribution of type II CRISPR-Cas Systems in Agricultural and Nonagricultural Campylobacter coli and Campylobacter jejuni isolates correlates with lack of shared environments. Genome Biol Evol. 2015;7: 2663-79. https://doi.org/10.1093/gbe/evv174.

31. Gundogdu O, da Silva DT, Mohammad B, Elmi A, Wren BW, van Vliet AHM, et al. The Campylobacter jejuni oxidative stress regulator RrpB is associated with a genomic Hypervariable region and altered oxidative stress resistance. Front Microbiol. 2016;7:2117. https://doi.org/10.3389/fmicb.2016.02117.

32. Dwivedi R, Nothaft $H$, Garber J, Xin Kin L, Stahl M, Flint A, et al. L-fucose influences chemotaxis and biofilm formation in Campylobacter jejuni. Mol Microbiol. 2016;101:575-89. https://doi.org/10.1111/mmi.13409.

33. Henderson IR, Navarro-Garcia F, Desvaux M, Fernandez RC, Ala'Aldeen D. Type $V$ protein secretion pathway: the autotransporter story. Microbiol Mol Biol Rev. 2004;68:692-744. https://doi.org/10.1128/MMBR.68.4.692-744.2004.

34. Chintoan-Uta C, Cassady-Cain RL, Stevens MP. Evaluation of flagellumrelated proteins FliD and FspA as subunit vaccines against Campylobacter jejuni colonisation in chickens. Vaccine. 2016. https://doi.org/10.1016/j. vaccine.2016.02.052.

35. Smart JP, Cliff MJ, Kelly DJ. A role for tungsten in the biology of Campylobacter jejuni: tungstate stimulates formate dehydrogenase activity and is transported via an ultra-high affinity $A B C$ system distinct from the molybdate transporter. Mol Microbiol. 2009;74:742-57. https://doi.org/10. 1111/j.1365-2958.2009.06902.x.

36. Bayliss CD, Palmer ME. Evolution of simple sequence repeat-mediated phase variation in bacterial genomes. Ann N Y Acad Sci. 2012;1267:39-44. https://doi.org/10.1111/j.1749-6632.2012.06584.x.

37. Ashgar SSA, Oldfield NJ, Wooldridge KG, Jones MA, Irving GJ, Turner DPJ, et al. CapA, an autotransporter protein of Campylobacter jejuni, mediates association with human epithelial cells and colonization of the chicken gut. J Bacteriol. 2007;189:1856-65. https://doi.org/10.1128/JB.01427-06.

38. Flanagan RC, Neal-McKinney JM, Dhillon AS, Miller WG, Konkel ME. Examination of Campylobacter jejuni putative Adhesins leads to the identification of a new protein, designated FlpA, Required for Chicken Colonization. Infect Immun. 2009;77:2399-407. https://doi.org/10.1128/IAI. 01266-08.

39. Sheppard SK, Didelot X, Jolley KA, Darling AE, Pascoe B, Meric G, et al. Progressive genome-wide introgression in agricultural Campylobacter coli. Mol Ecol. 2013;22:1051-64. https://doi.org/10.1111/mec.12162.

40. Berthenet $E_{1}$ Thépault $A$, Chemaly $M$, Rivoal $K$, Ducournau A, Buissonnière $A$, et al. Source attribution of Campylobacter jejuni shows variable importance of chicken and ruminants reservoirs in non-invasive and invasive French clinical isolates. Sci Rep. 2019;9:8098. https://doi.org/10.1038/s41598-01944454-2.

41. McCarthy ND, Gillespie IA, Lawson AJ, Richardson J, Neal KR, Hawtin PR, et al. Molecular epidemiology of human Campylobacter jejuni shows association between seasonal and international patterns of disease. Epidemiol Infect. 2012;140:2247-55. https://doi.org/10.1017/ S0950268812000192.

42. de Haan CPA, Llarena A-K, Revez J, Hänninen M-L. Association of Campylobacter jejuni metabolic traits with multilocus sequence types. App Environ Microbiol. 2012;78:5550-4. https://doi.org/10.1128/AEM.01023-12.

43. Sheppard SK, McCarthy ND, Jolley KA, Maiden MCJ. Introgression in the genus Campylobacter: generation and spread of mosaic alleles. Microbiology. 2011; 157(Pt 4):1066-74. https://doi.org/10.1099/mic.0.045153-0.
44. Skarp-de Haan CP, Culebro A, Schott T, Revez J, Schweda EK, Hänninen M-L, et al. Comparative genomics of unintrogressed Campylobacter coli clades 2 and 3. BMC Genomics. 2014;15:129. https://doi.org/10.1186/1471-2164-15-129.

45. Morley L, McNally A, Paszkiewicz K, Corander J, Méric G, Sheppard SK, et al. Gene loss and lineage-specific restriction-modification systems associated with niche differentiation in the Campylobacter jejuni sequence type 403 clonal complex. Appl Environ Microbiol. 2015;81:3641-7. https://doi.org/10. 1128/AEM.00546-15.

46. Feng Y, Chen Z, Liu S-L. Gene decay in Shigella as an incipient stage of host-adaptation. PLoS One. 2011;6:e27754. https://doi.org/10.1371/journal. pone.0027754.

47. Gurevich A, Saveliev V, Vyahhi N, Tesler G. QUAST: quality assessment tool for genome assemblies. Bioinformatics. 2013;29:1072-5. https://doi.org/10. 1093/bioinformatics/btt086.

48. Seemann T. Prokka: rapid prokaryotic genome annotation. Bioinformatics. 2014;30:2068-9. https://doi.org/10.1093/bioinformatics/btu153.

49. Hall TA. BioEdit: a user-friendly biological sequence alignment editor and analysis program for windows 95/98/NT. Nucleic Acids Symp Ser. 1999; http://jwbrown.mbio.ncsu.edu/JWB/papers/1999Hall1.pdf. .

50. van Vliet AHM, Kusters JG. Use of alignment-free Phylogenetics for rapid genome sequence-based typing of Helicobacter pylori virulence markers and antibiotic susceptibility. J Clin Microbiol. 2015;53:2877-88. https://doi.org/10. 1128/JCM.01357-15

\section{Publisher's Note}

Springer Nature remains neutral with regard to jurisdictional claims in published maps and institutional affiliations.
Ready to submit your research? Choose BMC and benefit from:

- fast, convenient online submission

- thorough peer review by experienced researchers in your field

- rapid publication on acceptance

- support for research data, including large and complex data types

- gold Open Access which fosters wider collaboration and increased citations

- maximum visibility for your research: over $100 \mathrm{M}$ website views per year

At BMC, research is always in progress.

Learn more biomedcentral.com/submissions 